\title{
Derivation of Nanocellulose from Native Rice Husk
}

\author{
Md. Iqbal Hossain", Humayra Zaman, Taslima Rahman \\ Department of Chemical Engineering, Bangladesh University of Engineering and Technology, Dhaka 1000. Article Info: \\ Submitted on February 10, 2018, Accepted on May 30, 2018.
}

\begin{abstract}
Nanocellulose has been in numerous applications and can be obtained from bioresources. This work demonstrates the derivation of nanocellulose from an alternative option i.e. rice husk. The processed rice husk was refined by chemical and mechanical treatments. Nanocellulose was subsequently derived from the refined rice husk through acid hydrolysis followed by centrifugation, dialysis and ultrasonic treatment. Scanning Electron Microscopy ensured the nanoscale diameter while Fourier Transformed InfraRed Spectroscopy confirmed the removal of noncellulosic materials. It is therefore proposed that the native rice husk can also be utilized for manufacturing nanocellulose reducing its adverse environmental impacts.
\end{abstract}

Keywords: Nanomaterials; Nanocellulose; Bioresource; Biomass; Rice Husk.

\section{Introduction}

Application of nanomaterials has been increasing gradually. One of the smart nanomaterials is nanocellulose $\mathrm{e}^{1,2}$. Nanocellulose offers various advantages such as nanoscale dimension, high tensile strength and Young's modulus, high surface-to-volume ratio, lower thermal expansion coefficient, and unique optical properties, etc ${ }^{3-6}$. Nanocellulose is also renewable, biodegradable, and nontoxic ${ }^{4-6}$. Nanocellulose is therefore used in pharmaceuticals, electronics, membranes, papers, and food industries ${ }^{7}$.A variety of bioresources have already been investigated as the feedstock to derive nanocellulose ${ }^{8}$.

Rice is the largest cereal crop in the world. It is the staple food for over half the world's population. The rice milling industries generate a huge quantity of rice husk during the paddy milling process from the fields. It accounts for $20 \%$ of the 500 million tons of paddy produced worldwide ${ }^{9,10}$. Rice husk is considered as one of the most widely available agricultural wastes in many rice producing countries of the world. The rice milling industries sometimes dispose the rice husk through open burning process. This situation leads to environmental concerns and becomes a great environmental threat causing damage to the land and the surrounding area in which it was dumped. A number of potential applications for rice husk have been indicated in literature ${ }^{11}$. However husk have also proven to be a difficult feedstock for gasification and fluidized bed combustion ${ }^{9,12}$. This is due to the high ash content resulting in carbon conversion inefficiency. The rice husk can also undergo combustion to produce rice husk ash that is rich in silica. Hence there is still a growing demand for exploring more useful applications for rice hush. The approximate cellulose content of rice husk are 25$35 \%$. An alternative approach to utilize rice husk

*Corresponding Author: Md. Iqbal Hossain

Email: iqbalhossain@che.buet.ac.bd, Fax: (880) 2-966-5609 involves the exploitation of its cellulose content. Rice husk can be a promising feedstock for nanocellulose. The present study is therefore aimed to derive nanocellulose from rice husk.

\section{Experimentation}

Mechanical and chemical processes are reported in literature ${ }^{5,13-18}$. The synthesis scheme consisting of both mechanical and chemical processes used in this study to derive nanocellulose from native rice husk is shown in Figure 1 and described below ${ }^{17,18}$.

\section{Feedstock Processing}

Rice husk (RH) was washed rigorously with stirring at room temperature by distilled water to remove foreign materials. The washed RH was dried and stored in hermetic plastic container to prevent microbial attack. It was subsequently grinded to obtain fine particles. A soxhlet apparatus was used to remove wax/oil from the RH fine particles.

\section{Chemical Treatments}

After dewaxing the fine particles were stirred with 3.0$3.5 \mathrm{w} / \mathrm{V} \% \mathrm{KOH}$ aqueous solution at a weight ratio of 1: $12-15$ and boiled for 30 minutes, and the mixture was rested overnight. The filtrate was washed twice with distilled water and $10 \mathrm{v} / \mathrm{V} \% \mathrm{HCl}$ was added (100 $\mathrm{mL})$. The formed precipitate of silica was separated from organic residue. The lignocellulosic residue was then treated with 0.7-1.7 w/V \% Sodium Chlorite $\left(\mathrm{NaClO}_{2}\right)$ at a ratio of 1: $50-80 \mathrm{~g}$ solid/mL liquor for bleaching. It was kept boiling for 2 hours and checked constantly for $\mathrm{p}^{\mathrm{H}}$ to keep at 4 . The remaining solid was first treated with 5-10 w/V \% Sodium Bisulphite solution at room temperature for 1 hour using a solid to liquor ratio of $1 \mathrm{~g} / 50 \mathrm{~mL}$. It was then washed with distilled water and kept for drying at room temperature (CBangladesh Uni. of Engg.\&Tech 19 


\section{Chemical Engineering Research Bulletin 20(2018) 19-22}

until constant weight was reached. It was subsequently treated with 17.5 w/V\% Sodium Hydroxide $(\mathrm{NaOH})$ solution at room temperature for 8 hours using a solid to liquor ratio of $1 \mathrm{~g} / 50 \mathrm{~mL}$ and was washed and dried again as reported in the previous step.

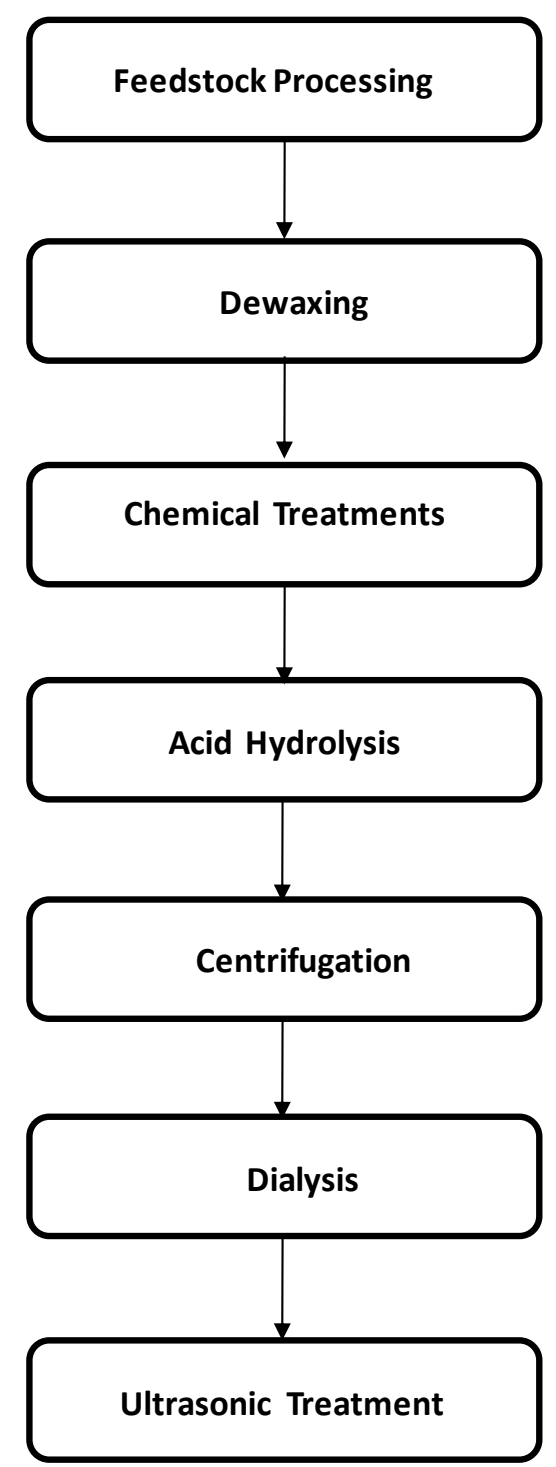

Figure 1: Demonstration of the derivation of nanocellulose from rice husk and rice straw.

\section{Formation and Isolation of Nanocellulose}

The nanocellulose was then formed by the acid hydrolysis of the dried cellulose intermediate. The acid hydrolysis was conducted using $64 \mathrm{wt} \%$ sulfuric acid solution for 30 minutes at continuous stirring condition. The entire process was carried out slowly on iced system to ensure that the nanocellulose particles were not burnt out by hot sulfuric acid. Then the solution was heated at $60^{\circ} \mathrm{C}$ temperature for around 15 minutes to achieve uniform concentration. It was washed with distilled water for diluting the acid concentration until $\mathrm{p}^{\mathrm{H}}$ was neutral.
After acid hydrolysis, the suspension was diluted to stop the reaction and allowed to settle for several hours until the suspensions were layered. The clear top layer was decanted off and then repeatedly washed with distilled water until they were not layered more. The suspension was then transferred into centrifuge tubes and centrifugation was performed repeatedly at 4000 rpm for 10-15 minutes to remove excess acid and water-soluble fragments. The fine particles were taken for further centrifugation to separate the nanocellulose suspension. The suspension was then washed with deionized water. The process was repeated several times to eliminate the acid content.

The resulting suspension was placed in the dialysis tubes having a molecular weight cutoff of 1200014000 and dialyzed against deionized water for several days until the water $\mathrm{p}^{\mathrm{H}}$ remained constant. The nanocellulose suspension was finally sonicated for 1530 minutes using an ultrasonic processor at $20-25 \mathrm{kHz}$ to isolate the nanocellulose. The ultrasonic treatment was carried out in an ice bath; the ice was maintained throughout the entire ultrasonication to avoid overheating.

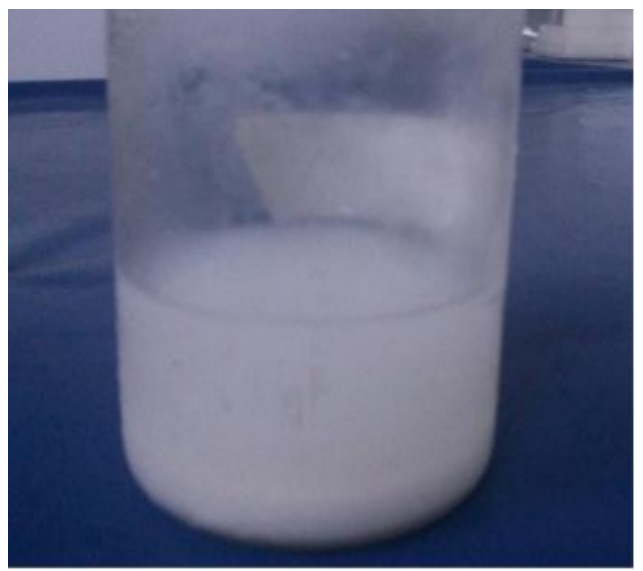

(a). Dispersed Nanocellulose

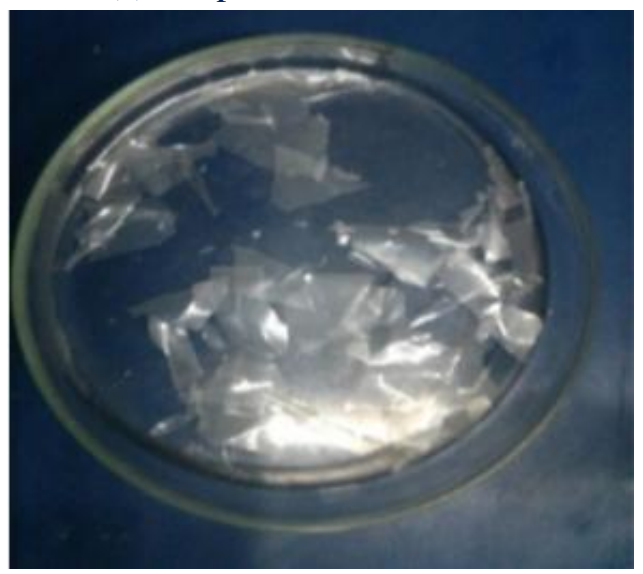

(b). Nanocellulose film

Figure 2: Demonstration of derived final cellulose and prepared film. 


\section{Chemical Engineering Research Bulletin 20(2018) 19-22}

\section{Preparation of Nanocellulose Film}

The suspension of nanocellulose after being stored in a refrigerator at $5^{\circ} \mathrm{C}$ for 2 days were poured into polystyrene Petri dishes, which were then kept in daylight for slow water evaporation. Solid thin films were obtained after the evaporation of water. All the films were kept in desiccators for characterization.

\section{Characterization of Nanocellulose}

The morphology of the prepared nanocellulose was tested through Scanning Electron Microscopy (SEM). SEM micrographs of the nanocellulose surface were taken using the TESCAN VEGA scanning electron microscope operated at $15 \mathrm{kV}$. The samples were coated with gold to eliminate the electron charging effects. Fourier Transformed Infrared Spectroscopy was also used to test the prepared nanocellulose. Nanocellulose samples were grinded in a ball mill, and the powders were pressed with $\mathrm{KBr}$ and analyzed in a Mattson Genesis II spectrometer at a resolution of 2 cm-1, 16 scans were performed over each sample.

The derived cellulose dispersion and prepared film are shown by Figure 2 (a) and (b), respectively.

\section{Results and Discussion}

Scanning Electron Microscopy was applied to determine the size of derived celluloses. SEM image of cellulose is shown in Figure 3. It was found from the size analyses of a series SEM images that the diameters of celluloses obtained from rice husk are 60$100 \mathrm{~nm}$.

It is known that the organic component of rice husk is composed of cellulose, hemicellulose, lignin and waxes, which most likely consist of alkene, esters, aromatics, ketones and alchohols, with different oxygen-containing functional groups, $\mathrm{OH}$ stretching (3650 to $3200 \mathrm{~cm}^{-1}$ ) and $\mathrm{C}=\mathrm{O}$ streching hemicelluloses $\left(1732 \mathrm{~cm}^{-1}\right)^{4-7}$. Some characteristic peaks of cellulose in $\mathrm{RH}$ can also be observed at $1640 \mathrm{~cm}^{-1}(\mathrm{OH}$ bending of absorbed water), $1420 \mathrm{~cm}^{-1}$ (CH2 strain), and 1375 and $1270 \mathrm{~cm}^{-1}$ ( $\mathrm{CH}$ bending). On the other hand, the vibrations of aromatic rings can be seen at 1606 and $1515 \mathrm{~cm}^{-1}$ (aromatic ring vibrations), which can only be related to lignins. The inorganic part is characterized by three bands associated to $\mathrm{Si}-\mathrm{O}-\mathrm{Si}$ bonds (situated at frequencies of 1100-1070, 799 and $\left.465^{\mathrm{cm}-1}\right)^{4-7}$.

A FTIR spectrum of nanocellulose (NC) from rice husk is presented in Figure 4. Characteristic cellulose peak was observed from the spectrum at 1375. The presence of small peaks at 1606 and $1515 \mathrm{~cm}^{-1}$ indicates that aromatic residues were present even in the NC. Non-cellulosic materials were absent in the spectrum.
The color of the untreated rice husk is brown and changes to brownish-orange after alkaline treatment. The bleached material is clearly different and appears completely white. These color changes are due to the removal of non-cellulosic materials and other impurities such as lignin, hemicelluloses, pectin, and wax upon chemical treatment. The observed inherent characteristic color as shown in Figure 2 (a) itself is an indication of the purity of derived cellulosic material.

Study to generate quantitative data for determining the percentage yield for nanocellulose is under progress.

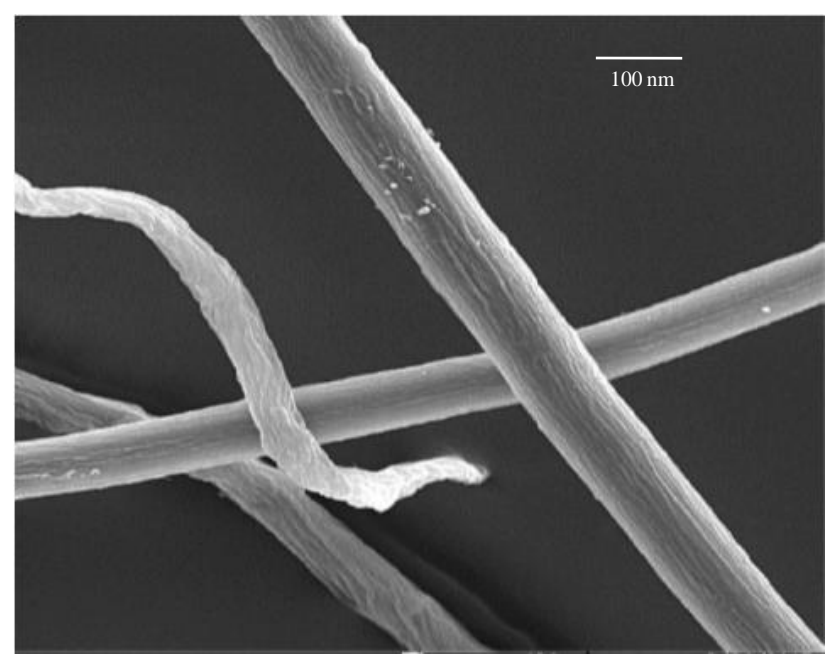

Figure 3: SEM image (Scale: $100 \mathrm{~nm}$ ) of nanocellulose obtained from rice husk.

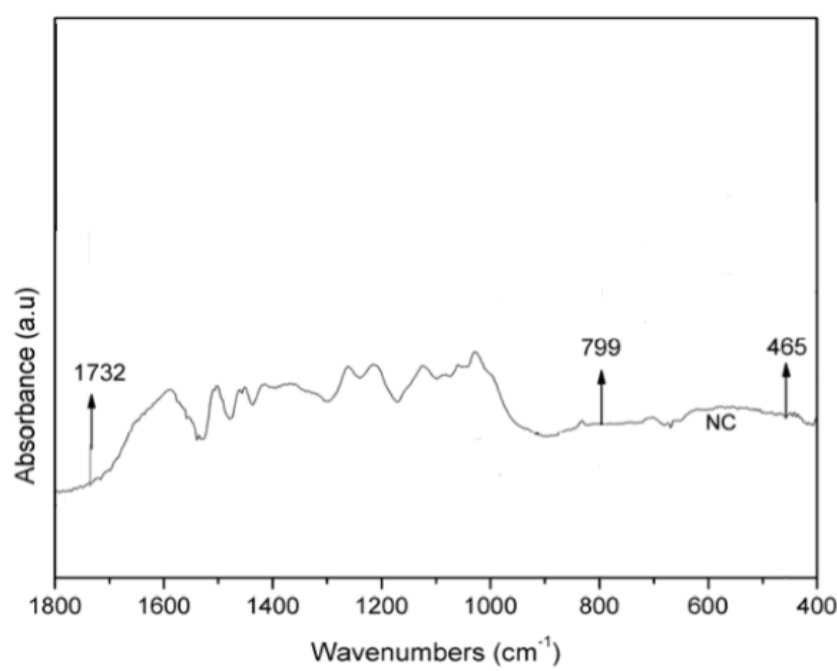

Figure 4: FTIR spectra of nanocellulose obtained from rice husk.

\section{Conclusion}

This study proposes an alternative use of rice husk as the feedstock for nanocellulose. The rice husk was first processed and refined through chemical and mechanical treatments. The former treatment was efficient in removing hemicellulose while the latter 


\section{Chemical Engineering Research Bulletin 20(2018) 19-22}

fully removed lignin. Nanocellulose was successfully derived from the refined rice husk through acid hydrolysis followed by centrifugation, dialysis and ultrasonic treatment. The diameter of nanocellulose ranged from 60-100 nm. Scanning Electron Microscopy ensured the diameter, while Fourier Transformed InfraRed Spectroscopy analysis confirmed the removal of non-cellulosic materials. Indigenous rice husk can therefore be considered alternatively for the manufacture of nanocellulose. However additional studies are required for determining the yield of nanocellulose and optimizing the derivation scheme.

\section{Acknowledgement}

Supports from Department of Chemical Engineering and Department of Chemistry, Bangladesh University of Engineering and Technology are gratefully acknowledged.

\section{References}

1. Hubbe, M. A., Rojas, O. J., Lucia, L., and Sain, M. Cellulosic nanocomposites: A review. Bioresources 3(3), 929-980, 2008.

2. Ioelovich, $\mathrm{M}$. Cellulose as a nanostructure polymer: A short review. Bioresources 3(4), 14031418, 2008.

3. Abe, K., Iwamoto, S., and Yano, H. S. Obtaining cellulose nanofibers with a uniform width of 15 $\mathrm{nm}$ from wood. Biomacromolecules 8(10), 32763278, 2009.

4. Eichhorn, S. J., Dufresne, A., Aranguren, M., Marcovich, N.E., Capadona, J. R., Rowan, S. J., Weder, C., Thielemans, W., Roman, M., Renneckar, S., Gindl, W., Veigel, S., Keckes, J., Yano, H., Abe, K., Nogi, M., Nakagaito, A.N., Mangalam, A., Simonsen, J., Benight, A. S., Bismarck, A., Berglund, L.A., and Peijs, T. Review: Current international research into cellulose nanofibres and nanocomposites. Journal of Material Science, 45, 1-33, 2010.

5. Habibi Y, Lucia LA, Rojas OJ. Cellulose nanocrystals: chemistry, self-assembly, and applications. Chemical Reviews, 110:3479-3500, 2010.

6. Siro' I, Plackett D. Microfibrillated cellulose and new nanocomposite materials: a review. Cellulose, 17:459-494, 2010.

7. Siqueira G, Bras J, Dufresne A, Cellulosic bionanocomposites: a review of preparation, properties and applications. Polymers, a, 2:728765, 2010.

8. Mohanty AK, Misra M, Hinrichsen G. Biofibres, biodegradable polymers and biocomposites: an overview. Macromolecular Material and Engineering, 276-277:1-24, 2000.

9. Bharadwaj, A., Wang, Y., Sridhar, S., and Arunachalam, V. S. Pyrolysis of rice husk. Current Science, 87(7), 981-986, 2004.

10. Sumithra Muthayya, Jonathan D. Sugimoto, Scott Montgomery, and Glen F. Maberly. An overview of global rice production, supply, trade, and consumption. Annals of the New York Academy of Sciences, ISSN 0077-8923, 1324, 7-14 C, 2014.

11. PothulaLalithaKumari, N. Sreenivasulu, P. Dinesh Sankar Reddy. Synthesis of nanocellulose fibers from various natural resources and residues. International Journal of Advance Research in Science and Engineering, 5, 1, 364-372, 2016.

12. Rozainee M, Ngo S.P, Salema A.A. Effect of fluidising velocity on the combustion of rice husk in a benchscale fluidised bed combustor for the production of amorphous rice husk ash. Bioresource Technology, 99, 703-713, 2008.

13. Araki, J., Wada, M., Kuga, M., and Okano, T. Birefringent glassy phase of a cellulose microcrystal suspension. Langmuir, 16(6), 24132415, 2000.

14. Iwamoto, S., Kai, W., Isogai, A., \& Iwata, T. Elastic modulus of single cellulose microfibrils from tunicate measured by atomic force microscopy. Biomacromolecules, 10(9), 25712576, 2009.

15. Jonoobi M, Harun J, Shakeri A, Misra M, Oksmand K.Chemical composition, crystallinity, and thermal degradation of bleached and unbleached kenaf bast (Hibiscus cannabinus) pulp and nanofibers. Bioresources, 4:626-639, 2009.

16. Dufresne A, Cavaille' JY, Vignon MR (1997) Mechanical behavior of sheets prepared from sugar beet cellulose micro fibrils. Journal of Applied Polymer Science, 64:1185-1194, 1997.

17. Iwamoto S, Nakagaito AN, Yano H, Nogi $M$. Optically transparent composites reinforced with plant fiber-based nanofibers. Applied Physics A, 81:1109-1112, 2005.

18. Eldho Abraham, B. Deepa, L.A. Pothen, J. Cintil, S. Thomas, M.J. John, R. Anandjiwala, S.S Narine. Environmental friendly method for the extraction of coir fiber and isolation of nanofiber. Carbohydrate polymers, 92, 1477-1483, 2013.

Publisher: Department of Chemical Engineering, Bangladesh University of Engineering and Technology (BUET). Review \&Publication: A submitted original manuscript is taken into review only if the uniqueness is found to be more than $85 \%$ in plag-scanning and selected for publication by the complete acceptance from at least two reviewers out of three. Home Page: http://www.banglajol.info/index.php/CERB. Indexed by Chemical Abstract Service (CAS), CEABA-VtB, Google Scholar, Scopus and DOAJ. 